\title{
Harvey Cushing's management of neurogenic thoracic outlet syndrome
}

\author{
Courtney Pendleton, MD, ${ }^{1}$ Allan J. Belzberg, MD, ${ }^{2}$ Robert J. Spinner, MD, ${ }^{3}$ and \\ Alfredo Quinones-Hinojosa, MD ${ }^{4}$
}

\begin{abstract}
1Department of Neurosurgery, Thomas Jefferson University Hospitals, Philadelphia, Pennsylvania; ${ }^{2}$ Department of Neurosurgery, The Johns Hopkins University School of Medicine, Baltimore, Maryland; ${ }^{3}$ Department of Neurosurgery, Mayo Clinic, Rochester, Minnesota; and ${ }^{4}$ Department of Neurosurgery, Mayo Clinic, Jacksonville, Florida
\end{abstract}

\begin{abstract}
Harvey Cushing is widely regarded as one of the forefathers of neurosurgery, and is primarily associated with his work on intracranial pathology. However, he had a clinical and academic interest in peripheral nerve surgery. Through the courtesy of the Alan Mason Chesney Medical Archives, the surgical records of the Johns Hopkins Hospital from 1896 to 1912 were reviewed. The records of a single patient undergoing brachial plexus exploration and cervical rib resection were selected for detailed review. The operative report and accompanying illustrations demonstrate Cushing's interest in adding approaches to the pathology of the brachial plexus to his operative armamentarium.
\end{abstract}

https://thejns.org/doi/abs/10.3171/2017.9.JNS17173

KEYWORDS Harvey Cushing; peripheral nerve; thoracic outlet syndrome; history

$\mathrm{T}$ HORACIC outlet syndrome (TOS) remains a challenging entrapment syndrome to diagnose and treat, despite description of its symptoms dating back centuries. Neurogenic TOS, caused by compression of the lower trunk of the brachial plexus, is known to physicians and surgeons interested in the peripheral nervous system.

Although descriptions of cervical ribs and their contributions to brachial plexus compression and TOS have been described since Vesalius, ${ }^{12}$ with whom Dr. Harvey Cushing (1869-1939) was most certainly familiar, cervical rib resection for treatment of symptoms remained a relatively rare occurrence, with only a few dozen cases recorded in the literature of the time.

Cushing, best known for his intracranial work, had an interest in surgery of the peripheral nerves, although his own reports of these cases were limited. Cushing's habit of bringing patient records from one institution to another throughout his career makes an accurate cataloguing of his peripheral nerve cases challenging, but retrospective review of his clinical records by ourselves and other research groups ${ }^{11}$ has demonstrated multiple operations for decompression, nerve repair, and reconstruction (including nerve transfer) in both pediatric and adult patients. In this paper we describe a patient who presented at the Johns Hopkins Hospital in 1902 and was diagnosed with motor and sensory disturbances caused by compression of the lower trunk of the brachial plexus by a cervical rib.

\section{Methods}

Following IRB approval, and through the courtesy of the Alan Mason Chesney Medical Archives, the surgical files of the Johns Hopkins Hospital from 1896 to 1912 were reviewed, spanning Cushing's career as a trainee and attending physician. Patients operated on by Dr. Cushing were selected. From these records, patients undergoing brachial plexus exploration were selected, and the records of a single patient undergoing exploration of the brachial plexus and resection of a rudimentary cervical rib for symptoms consistent with TOS were reviewed in detail.

\section{Case Report}

A 19-year-old woman presented to the neurology clinic with 3 months of right-hand intrinsic weakness and paroxysmal radicular pain. Atrophy was noted along the the- 
nar and hypothenar eminence. Examination with galvanic current failed to produce movement in the right-hand muscle groups. In addition, she complained of intermittent numbness throughout the right hemibody, with right lower extremity weakness, although this did not prevent her independent ambulation. She had no reported bowel symptoms, but frequent micturition dating to complications from pregnancy and delivery 3 years prior at another institution. She had undergone treatment with potassium iodide and outpatient galvanic current stimulation. Given the constellation of symptoms, Cushing was consulted to assess for spinal cord tumor or syringomyelia.

Cushing's physical examination notes document right hand and forearm atrophy, and sensory maps delineate the right upper extremity sensory changes and hypesthesias in the ulnar distribution, with patchy sensory changes in the torso and right leg. He notes "the entire brachial plexus above the clavicle is tender," and "On deep palpation above the clavicle $5 \mathrm{~cm}$ outside of sterno clavic joint is a distinct bony prominence not felt on other side." A radiograph taken at this time documented a cervical rib. ${ }^{10}$

The patient was brought to the operating room August 9, 1902. Figs. 1-3 provide Cushing's illustrations of the exposure.

Incision made as per diagram [Fig. 1].

Flap turned back. Contents of triangle, after fibers of omohyoid [cleared out] as in gland of neck operation, exposing [unclear ...] of jugular and subclavian veins. Part of sternomastoid divided, plexus well exposed. Scalenus anticus and phrenic [illegible] and turned forward c retraction.

Plexus well exposed in entirety [Fig. 1]. Large artery below with almost vertical course supposed to be [...] subclavian. Artery turned forward. It wound over 1 st rib behind scalenus anticus.

A dense fascial band was then exposed running longitudinally and winding around the lower part of the plexus [Fig. 2].

This fascial band seemingly was the anterior border of the Scalenus medius. It passed from what was subsequently found to be the tip of the rudimentary rib to the 1 st rib. It was well exposed by retracting the plexus upward and out and the artery downward and in.

Unfortunately for the operation the x-ray had shown a well devel. cerv. rib on the right and a rudimentary one on the left, and so considerable time was spent in searching for what was prob. on the other side of the neck viz a fully devel c. rib. In this search the 1st rib was well exposed and could have been excised $[. .$.$] Retraction of artery and plexus gave much room$ [...] It was finally realized that it was 1 st thoracic rib and search higher up then revealed the rudimentary sharp pointed rib high up in the neck from whose lip the dense fascial band (scalenus medius) wound around the lower cord of the plexus.

The plexus was then retracted downward and the rib excised after division of the dense band.

There was consid bleeding from the depth of the wound where the rib had been excised [Fig. 3] [...] All the trunks must have been consid. stretched. It was surprising to see how high in the neck the rib reached.

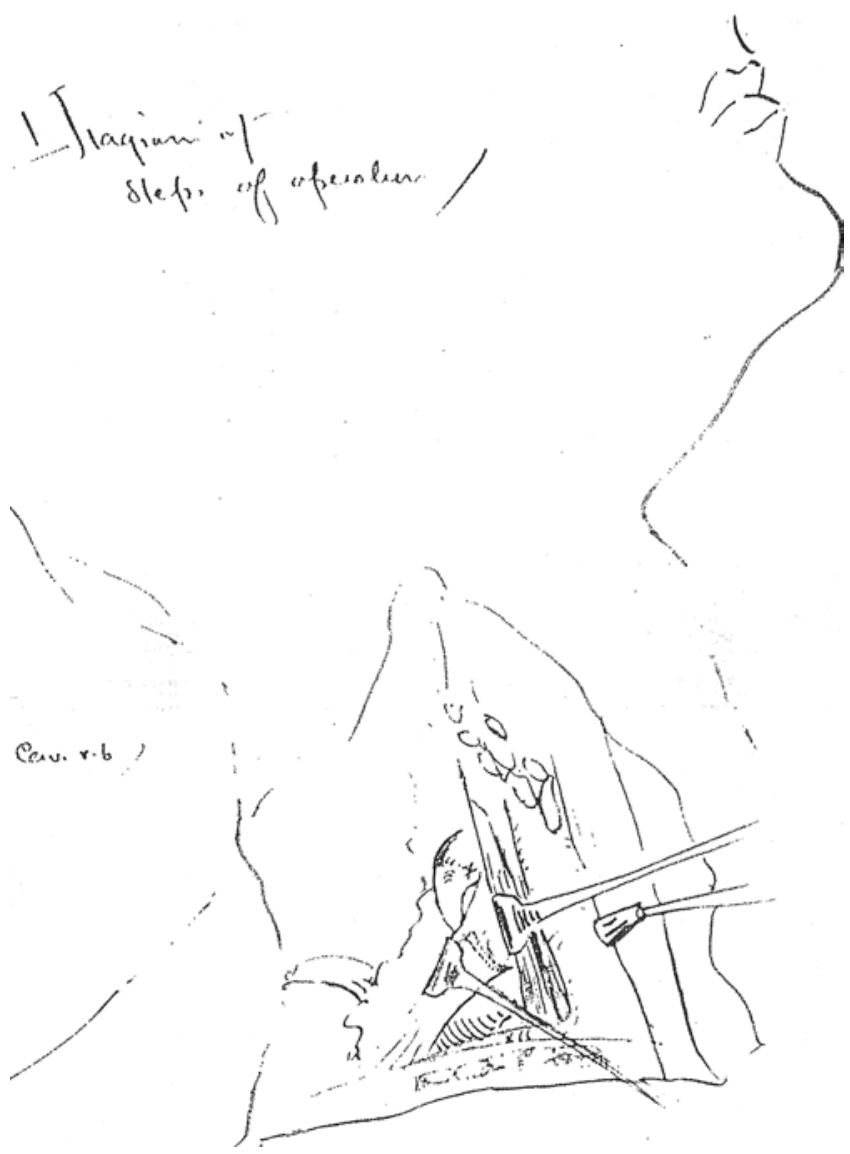

FIG. 1. Cushing's illustration of the exposure of the supraclavicular brachial plexus. Courtesy of The Alan Mason Chesney Medical Archives of The Johns Hopkins Medical Institutions.

In the immediate postoperative period, the patient experienced worsened sensory loss in the right upper extremity, which Cushing attributed to intraoperative stretching of the brachial plexus. By postoperative day 6 those symptoms had resolved, and Cushing's note documents "there is a distinct diminution (possibly disappearance) of rt sided hemianesthesia. Pain...has subsided." Unfortunately,

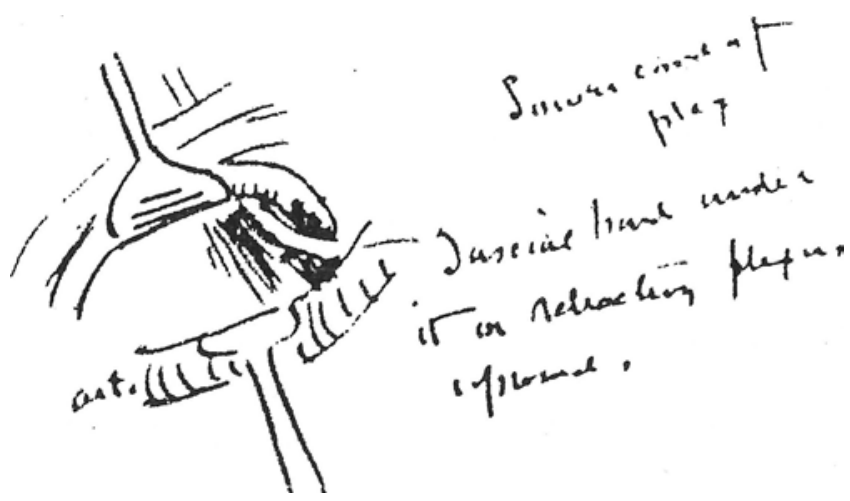

FIG. 2. Diagram of fascial band between cervical rib and scalenes compressing the lower trunk. Cushing's legend reads "Fascial band under it on retracting plexus exposed." Courtesy of The Alan Mason Chesney Medical Archives of The Johns Hopkins Medical Institutions. 


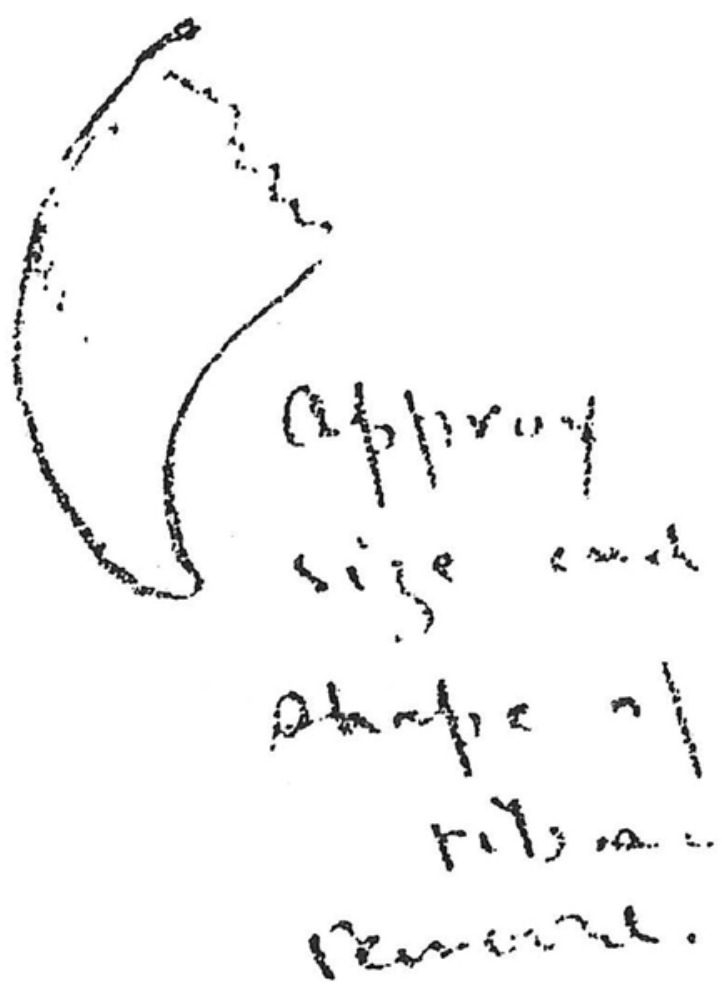

FIG. 3. Cushing's legend reads "approx size and shape of rib as removed." Courtesy of The Alan Mason Chesney Medical Archives of The Johns Hopkins Medical Institutions.

Cushing's last follow-up note states "Feby 141903 motor palsy has not cleared up."

\section{Discussion}

Here we describe the presentation and operative treatment of a patient with neurogenic TOS, which appears to be a novel intervention in Cushing's early career. The role of a cervical rib in the development of compressive symptoms would not have been foreign, given both Cushing's reading on the subject, and the research of his mentor and colleague William Halsted (1852-1922), who published research regarding the role of the cervical rib in changes to the subclavian artery. ${ }^{6}$

The medical record contains Cushing's own notations regarding anatomical studies describing the morphology of cervical ribs and their relation to structures in the supraclavicular region. ${ }^{3}$ In Cushing's discussion of this case in the Bulletin of the Johns Hopkins Hospital, he alludes to a "recent member of the Hospital Staff who was particularized by the presence of a cervical rib."10 The staff member, a young Lewellys Barker (1867-1943), began his career in William Osler's (1849-1919) clinic, and continued in Osler's footsteps to become the physician-in-chief at Johns Hopkins with a special interest in neurology. Given the immense support Osler played in developing Cushing's clinical skills and interest in the surgical treatment of neurological conditions, it is likely Cushing and Barker's paths intersected during their early training. Regardless, Barker's detailed treatise on the sensory manifestations of his cervical rib appears well known to Cushing, and the sensory distribution he reported mimics that illustrated in the patient described here. ${ }^{1}$

The patient examination documented by Cushing reflects hypesthesia in ulnar and medial antebrachial cutaneous nerve distribution, in addition to marked thenar and hypothenar eminence atrophy. This constellation of symptoms and findings is a hallmark of the Gilliatt-Sumner hand, associated with neurogenic TOS.

The patient described here also complained of sensory changes in her right hemibody, with occasional functional weakness in the right leg. These symptoms were intermittently elicited on physical examination, and although not documented in the chart, a discussion of the case in the Bulletin of the Johns Hopkins Hospital expresses belief that those symptoms "taken with the history of convulsive attacks and other neurotic manifestations, made it evident that we had to do with the combination of a definite local lesion (pressure on the lower part of the brachial plexus from the cervical rib) and a functional or hysterical condition." ${ }^{\prime \prime}$ In the same discussion, Cushing cited a case report describing a patient with a cervical rib and similar symptoms, with a hypothesis that the lower extremity symptoms may be attributable to spinal gliosis, which might be a congenital anomaly concurrent with a supernumerary rib. ${ }^{10}$ These assumptions were within the bounds of medical knowledge of the time. Given the resolution of the patient's sensory symptoms immediately postoperatively, Occam's razor may be dulled in this instance, i.e., the patient's symptoms may be attributable to multiple causes, one of which was operatively treated.

Although the technology at the turn of the 20th century was less advanced, Cushing's notes document use of roentgenography as part of the patient's evaluation, which is unsurprising given his extensive use of x-rays in diagnosis and operative planning, beginning with his first published manuscript. ${ }^{4,5}$

Although surgery of the peripheral nerves remained a rudimentary endeavor among Cushing's contemporaries, the anatomical work of Santiago Ramón y Cajal (18521934) and his contemporaries had delineated the ability of injured nerves to sprout axons at the proximal stump, ${ }^{7,9}$ while clinical experiments with nerve anastomoses and grafting were reported to result in functional recovery. ${ }^{8,11}$ In this milieu, Cushing's foray into peripheral nerve entrapment management is anchored firmly in surgical and neurological interests of his peers. ${ }^{8,13}$ While Cushing's clinical interests were largely focused on intracranial pathology, he continued dabbling in peripheral nerve surgery well into his later career at Peter Bent Brigham Hospital, ${ }^{2,11}$ indicating he believed such operations were within the purview of a specialized neurological surgeon, and it is worth noting the contributions of Cushing, an early pioneer of neurosurgery, to the field of peripheral nerve entrapment syndromes.

\section{References}

1. Barker LF: A case of circumscribed unilateral and elective sensory paralysis. J Exp Med 1:348-360, 1896

2. Black PM, Moore MR, Rossitch E: The Surgical Art of Harvey Cushing. Park Ridge, IL: American Association of Neurological Surgeons, 1993 
3. Brush C: Notes on cervical ribs. Bull Johns Hopkins Hosp 12:114-117, 1901

4. Cushing H: Haematomyelia from gunshot wound of the cervical spine. Bull Johns Hopkins Hosp 8:195-197, 1897

5. Gunderman RB, Seymour ZA: Harvey W. Cushing. AJR Am J Roentgenol 194:296-298, 2010

6. Halsted WS: An experimental study of circumscribed dilation of an artery immediately distal to a partially occluding band, and its bearing on the dilation of the subclavian artery observed in certain cases of cervical rib. J Exp Med 24:271286, 1916

7. Harrison RG: The outgrowth of the nerve fibre as a mode of protoplasmic movement. J Exp Zool 9:787-847, 1910

8. Little KM, Zomorodi AR, Selznick LA, Friedman AH: An eclectic history of peripheral nerve surgery. Neurosurg Clin N Am 15:109-123, 2004

9. Ramón y Cajal S: Estudios sobre la degeneración y regeneración del sistema nervioso. Madrid: N. Moya, 1914

10. Thomas H, Cushing H: Exhibition of two cases of radicular paralysis of the brachial plexus. One from the pressure of a cervical rib with operation. The other of uncertain origin. Bull Johns Hopkins Hosp 14:315-319, 1903

11. Tubbs RS, Patel N, Nahed BV, Cohen-Gadol AA, Spinner RJ Reflections on the contributions of Harvey Cushing to the surgery of peripheral nerves. J Neurosurg 114:1442-1448, 2011
12. Urschel HC, Kourlis H: Thoracic outlet syndrome: a 50-year experience at Baylor University Medical Center. Proc Bayl

Univ Med Cent 20:125-135, 2007

13. Walker AE: A History of Neurological Surgery. New York: Hafner, 1967

\section{Disclosures}

The authors report no conflict of interest concerning the materials or methods used in this study or the findings specified in this paper.

\section{Author Contributions}

Conception and design: Pendleton. Acquisition of data: Pendleton. Analysis and interpretation of data: Pendleton. Drafting the article: Pendleton. Critically revising the article: all authors. Reviewed submitted version of manuscript: all authors. Approved the final version of the manuscript on behalf of all authors: Quinones-Hinojosa.

\section{Correspondence}

Alfredo Quinones-Hinojosa: Mayo Clinic, Jacksonville, FL. quinones-hinojosa.alfredo@mayo.edu. 